Ann. Zootech., I972, 21 (3), 333-345.

\title{
INFLUENCE DU TRAITEMENT HYDRO-THERMIQUE D'UNE ORGE IMMATURE OU MÛRE RÉHYDRATÉE, SUR SA VALEUR ALIMENTAIRE ET CELLE DE L'URÉE AJOUTÉE CHEZ LE JEUNE TAURILLON
}

\author{
S.-Z. ZELTER et Geneviève CHARLET-LERY \\ avec la collaboration de F. Le Deschault de Monredon, \\ Annick Eloy et Michèle FiszLewrcz \\ Laboratoire de Recherches sur la Conservation et l'Efficacité des Aliments, \\ Centre national de Recherches zootechniques, I. N.R. A., \\ 78 - Jouy-en-Josas

\section{RÉSUMÉ}

Une orge mûre, préalablement réhumidifiée à $36 \mathrm{p}$. Ioo a été soumise à un traitement hydrothermique à la pression atmosphérique, à une température relativement faible $\left(57^{\circ}-92^{\circ} \mathrm{C}\right)$ ou forte $\left(75^{\circ}-107^{\circ} \mathrm{C}\right)$, qui augmente sensiblement la richesse en amidon aisément dégradable in vitro par la $\alpha$-amylase. On a cherché à savoir si ce type de traitement améliorait comme il le fait avec l'orge immature, l'efficacité azotée de l'urée ajou tée et la croissance chez le jeune taurillon normand.

Le traitement faible et surtout fort de l'orge mûre abaisse notablement la digestibilité et la rétention azotée ainsi que la croissance. Cet effet négatif de la chaleur est hautement significatif et l'on suppose qu'il est essentiellement imputable à la dénaturation des proteines et à la réduction sensible constatée dans la céréale ainsi traitée, de la richesse en certains acides aminés indispensables, plus spécialement en lysine totale et disponible.

Par contre, l'orge mûre non traitée et la même orge immature déshydratée avec le traitement fort procurent des résultats absolument identiques.

\section{INTRODUC'TION}

Dans une précédente publication (ZELTER et $a l$, I97I), nous avons montré que le traitement hydrothermique d'une céréale (orge, maîs) récoltée au stade immature améliore très significativement, chez le taurillon en croissance, l'efficacité azotée de l'urée ajoutée qui acquiert une valeur équivalente à celle d'un tourteau de soja. L'action bénéfique du traitement se relierait à une très forte augmentation de la 
fraction d'amidon facilement attaquable par les $\alpha$-amylases, et à une accélération de la libération de son énergie dans le rumen. Cette libération deviendrait concomitante de 1'uréolyse, permettant ainsi aux microorganismes de la panse d'utiliser la plus grosse part du $\mathrm{N}-\mathrm{NH}_{3}$ formé pour leur propre protéinosynthèse (DURAND, I970; ZELTER et al., I97I).

Nous nous sommes dès lors posés la question de savoir si semblable traitement appliqué au grain mûr de céréale, préalablement réhumidifié, procurerait des résultats équivalents à ceux du grain immature (ZELTER et al., I97I).

Certains auteurs signalent cependant que la cuisson du grain d'orge mûr (Kojıma et al., I968) ou la gélatinisation par autoclavage du grain de maîs (MuDd et Perry, I969) occasionne une baisse sensible de la digestibilité de la plupart des éléments constitutifs majeurs de la matière organique.

Afin d'élucider ces deux points, nous avons observé chez des taurillons en croissance l'influence du traitement thermique d'une même orge immature ou mûre réhydratée, sur la digestibilité, l'efficacité azotée de l'urée ajoutée, le niveau d'ingestion et la croissance, l'orge mûre non traitée étant prise comme référence.

\section{MATÉRIEL E'T MÉTHODES}

\section{r. - Traitement de l'orge}

L'orge (variété Rika) a été récoltée en 1970 sur la même parcelle, d'abord au stade immature (I 7-7-1970) en coupe haute (épis entiers $+26 \mathrm{~cm}$ de paille supérieure) avec une récolteuse-hacheuse, puis au stade grain mûr (12-8-1970) séparément grain et paille avec une moissonneusebatteuse.

L'orge immature (OI), dont la teneur en humidité atteignait à la récolte 57,7 p. roo, a été aussitôt déshydratée à $13^{\circ} \mathrm{C}$ (température de l'air admis) pendant 30 minutes, dans un séchoir à tablier Scolari, et broyée à la grille $5 \mathrm{~mm}$.

Le grain d'orge mûve (teneur en humidité I2-I3 p. Ioo) a été divisé en trois lots. L'un (OM) n'a pas subi de traitement thermique. Les deux autres lots ont été réhydratés par macération dans l'eau durant 24 heures, ce qui a permis d'atteindre une teneur en humidité de $36 \mathrm{p}$. roo, et déshydratés immédiatement après dans le même appareil, pourvu à divers endroits du tablier de thermo-couples enregistreurs. Le lot $\mathrm{OMD}_{1}$ a subi un traitement thermique relativement faible (température moyenne de la masse en milieu et fin de séchage $: 57^{\circ}$ et $92^{\circ} \mathrm{C}$ ) et le lot $\mathrm{OMD}_{2}$ un traitement thermique relativement fort (température moyenne en milieu et fin de séchage $: 75^{\circ}$ et $\mathrm{I} 07^{\circ} \mathrm{C}$ ). Les températures respectives de l'air chaud admis s'élevaient à $\mathrm{I} 27^{\circ}$ et $\mathrm{I} 45^{\circ} \mathrm{C}$; l'épaisseur des couches du grain séché oscillait entre 6 et $8 \mathrm{~cm}$ et la durée de séchage du même ordre que celle de l'orge immature (environ 30 minutes).

Les trois lots de grains mûrs, traités ou non, ont été également broyés à la grille de $5 \mathrm{~mm}$, additionnés d'une quantité donnée de leur propre paille broyée, afin d'obtenir une teneur en cellulose brute comparable à celle de l'orge immature.

\section{2. - Dispositif expérimental}

\section{a) Animaux.}

L'expérience a été effectuée avec $\mathbf{I} 2$ taurillons normands dont l'âge variait entre 6 et to mois et le poids initial entre 160 et $200 \mathrm{~kg}$. Les animaux ont été répartis en 4 groupes $(\mathrm{A}, \mathrm{B}, \mathrm{C}, \mathrm{D}) \mathrm{de}$ 3 sujets, de poids et d'âge comparables.

La technique des blocs a été appliquée. Les animaux séjournaient en stalles individuelles à bilan. Deux périodes de bilan de digestibilité et de rétention azotée ont été effectuées successivement, chacune de ces périodes étant précédée d'une phase d'accoutumance de 4 semaines dont les trois premières en séjour au sol. 
La durée de chaque période de bilan était de 5 jours répartis en 3 sous-périodes hebdomadaires successives de 5 jours.

A l'issue de ces deux périodes de bilan, les sujets de chaque bloc (4 animaux) ont été soumis durant 12 semaines, en loges individuelles, à des mesures de consommation de nourriture (alimentation ad libitum) et de croissance, après avoir été réadaptés au séjour au sol, sur litière pendant I9 jours.

Lors des bilans, les animaux étaient pesés 3 jours consécutifs en début et fin de période, et tous les $\mathrm{r} 5$ jours pendant la période d'appétabilité-croissance.

\section{b) Régimes alimentaires et modalités de rationnement.}

Les 4 lots d'orge + paille ont été supplémentés par de l'urée ( $16-17 \mathrm{~g} / \mathrm{kg}$ de MS) afin d'élever la concentration de la matière sèche en matières azotées totales à environ $\mathrm{r} 3 \mathrm{p}$. Ioo, ce qui cor-

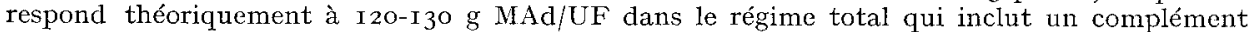
minéral (4 p. Ioo) adéquat $\left({ }^{2}\right.$ ) et de vitamines $\mathrm{A}$ et $\mathrm{D}_{3}$ (respectivement 2500 et $250 \mathrm{UL} / \mathrm{kg}$ d'aliment).

Chacun de ces 4 mélanges (OIDU-OMU-OMD $\mathrm{U}_{-}-\mathrm{OMD}_{2} \mathrm{U}$ ) a été ensuite aggloméré à la presse en bouchons de $7 \mathrm{~mm}$ de diamètre. Ces bouchons ont été distribués tels quels, accompagnés de paille grossière hachée ( $5 \mathrm{p}$. Ioo de la matière sèche totale de la ration) a fin d'éviter des troubles de rumination, sauf durant la période de croissance où les animaux consommaient librement la paille utilisée comme litière.

Schéma de succession des régimes*

\begin{tabular}{|c|c|c|c|}
\hline \multirow{2}{*}{ Groupes } & \multicolumn{2}{|c|}{$\begin{array}{c}\text { Périodes d'accoutumance } \\
\text { et de bilans }\end{array}$} & \multirow{2}{*}{$\begin{array}{c}\text { Période } \\
\text { " appétabilité - croissance " } \\
\text { Période III }\end{array}$} \\
\hline & $\begin{array}{l}\text { Période I } \\
(6 \text { sem. })\end{array}$ & $\begin{array}{l}\text { Période II } \\
(6 \text { sem. })\end{array}$ & \\
\hline $\begin{array}{l}\mathrm{A} \\
\mathrm{B}\end{array}$ & $\begin{array}{l}\text { OMU } \\
\text { OIDU }\end{array}$ & $\begin{array}{l}\text { OLDU } \\
\text { OMU }\end{array}$ & $\begin{array}{l}\text { OIDU } \\
\text { OMU }\end{array}$ \\
\hline $\begin{array}{l}\mathrm{C} \\
\mathrm{D}\end{array}$ & $\begin{array}{l}\mathrm{OMD}_{2} \mathrm{U} \\
\mathrm{OMD}_{1} \mathrm{U}\end{array}$ & $\begin{array}{l}\mathrm{OMD}_{1} \mathrm{U} \\
\mathrm{OMD}_{2} \mathrm{U}\end{array}$ & $\begin{array}{l}\mathrm{OMD}_{1} \mathrm{U} \\
\mathrm{OMD}_{2} \mathrm{U}\end{array}$ \\
\hline
\end{tabular}

* OID : orge immature déshydratée ; OM : orge mûre ; $\mathrm{OMD}_{1}$ : orge mûre réhumidifiée et déshydratée à faible température ; $\mathrm{OMD}_{2}$ : orge mûre réhumidifiée et déshydratée à forte température ; $\mathrm{U}$ : urée.

Les rations effectivement ingérées renfermaient par conséquent 56,7 p. Ioo d'orge, 38, I p. Ioo de paille d'orge, I,4 à I, 5 p. Ioo d'urée et 3,8 p. Ioo de complément minéral vitaminisé.

Afin de réduire au maximum les refus de nourriture, la quantité totale de matière sèche distribuée lors du séjour en stalle à bilan a été limitée à $80 \mathrm{~g}$ de matière sèche/ $/ \mathrm{kg} \mathrm{P}^{\mathbf{0}, 75}$; ce qui assurait, d'après nos expériences précédentes avec le même type d'animaux, l'entretien et un gain corporel journalier de $700 \mathrm{~g}$ (ZELTER et al., I971).

Les 4 régimes ont été permutés deux par deux d'une période de bilan à l'autre selon le schéma indiqué ci-dessus.

Par contre, pendant la période III (appétabilité-croissance) où les groupes ont conservé chacun leur régime de la période II, les animaux ont été alimentés individuellement ad libitum.

(1) Formule du mélange minéral: $\mathrm{CaCO}_{3}, 9,490$ p. xoo; $\mathrm{Ca}_{2} \mathrm{H}_{2}\left(\mathrm{PO}_{4}\right)_{2}, 55$ p. 100; $\mathrm{NaCl}, 30$ p. 100; $\mathrm{SO}_{4} \mathrm{Cu}$, ${ }_{5} \mathrm{H}_{2} \mathrm{O}, 0,06$ p. 100; $\mathrm{SO}_{4} \mathrm{Zn}, 7 \mathrm{H}_{2} \mathrm{O}, \mathrm{I}, 5$ p. 100; $\mathrm{SO}_{4} \mathrm{CO}$, $7 \mathrm{H}_{2} \mathrm{O}-0,010$ p. 100; $\mathrm{SO}_{4} \mathrm{Mn}, 7 \mathrm{H}_{2} \mathrm{O}$, o, 600 p. roo; $\mathrm{SO}_{4} \mathrm{Fe}$, $7 \mathrm{H}_{2} \mathrm{O}, 3,000$ p. IOO; $\mathrm{SO}_{4} \mathrm{Na}$, IO $\mathrm{H}_{2} \mathrm{O}$, o,340 p. IOO. 


\section{3. - Collecte, conservation et analyse des ingesta et excreta}

Les méthodes utilisées ont été décrites dans notre publication antéricure (ZELTER et al., I97 I). En outre, on a dosé sur tous les lots d'orge, avant et après traitement hydrothermique, l'amidon total selon Ewers et l'amiclon solubilisé in vitro par la $\alpha$-amylase selon GuILbot et Mercier (r962). On a déterminé également, sur les protéines de l'orge immature et mûre, avant et après ce traitement, la composition en acides aminés essentiels sur autoanalyseur Phoenix, la lysine clisponible selon RoAcH et al. $(1967)$, les tencurs en albumines - globulines et en prolamines.

\section{RÉSULTATS}

Les caractéristiques de 1'amidon et son comportement cinétique à la $\alpha$-amylase sont données dans le tableau I et la figure $\mathbf{I}$.

\section{TABLEAU I}

Caractéristique's de l'état de l'amidon

\begin{tabular}{|c|c|c|c|}
\hline & $\begin{array}{l}\text { Amidon total } \\
\text { p. } 100 \text { de MS }\end{array}$ & $\begin{array}{c}\text { yd'amidon } \\
\text { facilement } \\
\text { attaquables ì la } \\
\alpha \text {-amylase/kg } \\
\text { de MS }\end{array}$ & $\begin{array}{c}\text { Rapport amiclon } \\
\text { facilement } \\
\text { attaquable/urée } \\
\text { incorporée dans la } \\
\text { ration totale }\left({ }^{1}\right)\end{array}$ \\
\hline OII $*$ & 42,4 & 271 & 15,9 \\
\hline Ox ${ }^{*}$ & 39,6 & 107 & 6,7 \\
\hline $\mathrm{OMII})_{1} *$ & 38,7 & 132 & 8,3 \\
\hline $\mathrm{OMI}_{2} *$ & 40,3 & 185 & 11,6 \\
\hline
\end{tabular}

(1) Urće incorporée par $\mathrm{kg}$ de MS : ONIU, OMDO, $\mathrm{U}, \mathrm{OMD} \mathrm{D}_{2} \mathrm{U}=16 \mathrm{~g}$; urée incorporée par $\mathrm{kg}$ de MS : OIDU $=17 \mathrm{~g}$.

* OII) : orge immature deshydratée ; OM : orge mûre ; OMID : orge mûre traitement faible; $\mathrm{OMD}_{2}$ : orge nùre traitement fort.

Quel que soit le stade de récolte, la teneur en amidon total de l'orge (paille incluse) est similaire et du même ordre que celle enregistrée avec les orges immatures de nos précédentes expériences; mais la fraction d'amidon digérée à la $\alpha$-amylase est près de 3 fois plus faible dans l'orge mûre. Après le traitement hydrothermique de celle-ci, cette fraction s'accroît seulement de $23 \mathrm{p}$. Ioo à basse température et de $72 \mathrm{p}$. Ioo à forte température. Dans ce dernier cas, elle devient comparable à la concentration observée pour l'orge immature déshydratée de la récolte I968 (I 8,5 p. Ioo et $20,6 \mathrm{p}$. IOO).

Il s'ensuit des modifications du rapport amidon soluble/urée notables et différentes selon les régimes (tabl. I). 
La composition en acides aminés est donnée dans le tableau 2 et celles en lysine disponible et en certaines fractions azotées extractibles dans le tableau 3.

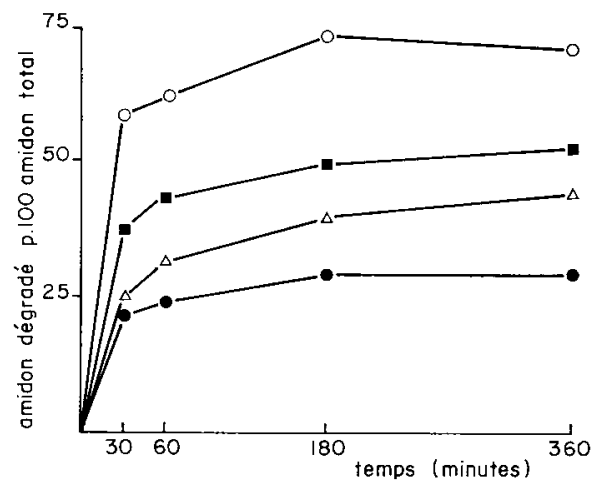

FIG. x. - Cinétique de l'amylolyse in vitro

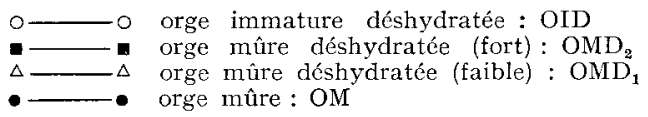

TABLEAU 2

Infuence de la déshydratation

sur la composition en acides aminés (en g/I6 $\mathrm{g} \mathrm{N}$ ) de l'orge müre

\begin{tabular}{|c|c|c|}
\hline Acides aminés & Orge mûre & $\begin{array}{l}\text { Orge déshydratée } \\
\text { (trajtement fort) }\end{array}$ \\
\hline Acide aspartique $\ldots \ldots$ & 6,8 & 6,0 \\
\hline Thréonine .......... & 3,85 & 3,55 \\
\hline Sérine $\ldots \ldots \ldots \ldots \ldots$ & 4,45 & 4,3 \\
\hline Acide glutamique $\ldots$. & 21,5 & 22,1 \\
\hline Proline $\ldots \ldots \ldots \ldots$ & 10,2 & 10,2 \\
\hline Glycine $\ldots \ldots \ldots \ldots$ & 4,3 & $t_{1}, 2$ \\
\hline Alanine $\ldots \ldots \ldots \ldots$. & 4,4 & 4,25 \\
\hline Valine $\ldots \ldots \ldots \ldots \ldots$ & 4,7 & 5,0 \\
\hline Isoleucine $\ldots \ldots \ldots \ldots$ & 3,45 & 3,15 \\
\hline Leucine $\ldots \ldots \ldots \ldots \ldots$ & 7,35 & 7,0 \\
\hline Tyrosine $\ldots \ldots \ldots \ldots$ & 3,2 & 3,1 \\
\hline Phénylalanine $\ldots \ldots \ldots$ & 5,0 & 4,95 \\
\hline Lysine $\ldots . . . . . .$. & 4,05 & 3,5 \\
\hline Histidine $\ldots \ldots \ldots \ldots$ & 2,2 & 2,0 \\
\hline Arginine............ & 5,9 & 5,1 \\
\hline Méthionine . . . . . . . & 1,7 & 1,7 \\
\hline Cystine............. & 2,3 & 2,2 \\
\hline
\end{tabular}

Les compositions des rations effectivement consommées figurent dans le tableau 4. Elles sont quasiment isocellulosiques (I4,8 à I6,9 de cellulose Weende p. Ioo de matière sèche) et isoazotées (I2,9 à I3, 3 p. IoO MS de matières azotées 
totales $(\mathrm{N} \times 6,25)$ dont 34 à 36 p. roo sont sous forme d'azote uréique). L'azote soluble est compris entre 35,5 et 39,7 p. Ioo d'azote total.

Par suite du rationnement limité, les gains moyens de poids réalisés en stalles à bilan ont été relativement faibles : la moyenne des groupes oscillait entre 530 et $6 \mathrm{I} 5 \mathrm{~g} /$ jour, la différences ne sont pas significatives.

\section{TABLEAU 3}

Teneur en certaines fractions azotées, en lysine tota?e et disponible (en g/I6 g N).

\begin{tabular}{|c|c|c|c|c|c|}
\hline & \multicolumn{3}{|c|}{ Orge mûre } & \multicolumn{2}{|c|}{ Orge immature } \\
\hline & $\begin{array}{l}\text { non } \\
\text { traitée }\end{array}$ & $\begin{array}{c}\text { traitement } \\
\text { fort }\end{array}$ & $\begin{array}{c}\text { traitement } \\
\text { faible }\end{array}$ & $\begin{array}{c}\text { non } \\
\text { traitée }\end{array}$ & traitée \\
\hline $\begin{array}{l}\text { Lysine totale } \\
\% \text { de chute }\end{array}$ & 4,05 & $\begin{array}{r}3,5 \\
-13,5 \%\end{array}$ & 一 & 4,79 & $\begin{array}{r}3,78 \\
-21 \%\end{array}$ \\
\hline $\begin{array}{l}\text { Lysine disponible } \\
\text { \% de chute }\end{array}$ & 3,77 & $\begin{array}{c}3,02 \\
-20,0 \%\end{array}$ & - & 4,55 & $\begin{array}{r}3,45 \\
-24 \%\end{array}$ \\
\hline $\begin{array}{c}\mathrm{N} \text { albumine }+ \text { globuline } \\
\% \text { de } \mathrm{N} \text { total }(\mathbf{1}) \\
\% \text { de chute }\end{array}$ & 26,8 & ${ }^{8,5}$ & $6 \%$ & 一 & - \\
\hline $\begin{array}{l}\mathrm{N} \text { prolamine } \\
\% \text { de } \mathrm{N} \text { total }(\mathbf{2}) \\
\% \text { de chute }\end{array}$ & 22,2 & $\begin{array}{r}8,1-6 \\
-6\end{array}$ & $3 \%$ & 一 & - \\
\hline
\end{tabular}

(1) Fraction azotée solubilisée par $\mathrm{NaCl} 0,5 \mathrm{M}$. Cette fraction contient aussi l'azote non protéique.

(2) Fraction azotée solubilisée par éthanol à $60 \%$ après élimination de NNP des albumines et des globulines.

\section{TABLEAU 4}

Composition des rations effectivement consommées en p. 100 de MS

\begin{tabular}{l|c|c|c|c|c}
\hline \hline & $\begin{array}{c}\text { Matières } \\
\text { organiques }\end{array}$ & $\begin{array}{c}\text { Matières } \\
\text { azotées }\end{array}$ & N urće \% N total & $\begin{array}{c}\text { Cellulose } \\
\text { Weende }\end{array}$ & $\mathrm{N}$ sol \% N total \\
\hline & 91,8 & 13,3 & 36,1 & 14,8 & 39,6 \\
OIDU* $_{\text {OMU* }}$ & 93,2 & 13,3 & $3 \%, 8$ & 16,9 & 39,7 \\
$\mathrm{OMD}_{1} \mathrm{U}^{*}$ & 93,3 & 13,2 & 34,0 & 15,9 & 35,5 \\
$\mathrm{OMD}_{2} \mathrm{U}^{*}$ & 93,4 & 12,9 & 35,0 & 16,8 & 35,9 \\
\hline
\end{tabular}

* OIDU : orge immature déshydratée + urée ; OMU : orge mûre + urée ; $O M D_{1} \mathrm{U}$ : orge mûre traitement faible + urée; $\mathrm{OMD}_{2} \mathrm{U}$ : orge mûre traitement fort + urée. 


\section{I. - Digestibilité et bilan d'azote}

Aucun trouble digestif ou métabolique n'a été observé durant les 7 mois d'expérience.

a) Niveau de consommation de matière sèche, utilisation de l'énergie et de l'azote (tab1. 5 et 6).

Grâce au rationnement égalitaire adopté, les refus de nourriture étaient négligeables et les niveaux de consommation de matière sèche $\left(\mathrm{MSi} / \mathrm{P}^{0,75}\right)$ pratiquement identiques dans tous les groupes avec tous les régimes (tabl. 5).

\section{TABLEAU 5}

Coefficients de digestibilité et valeur nutritive des régimes $\pm s m$

\begin{tabular}{|c|c|c|c|c|c|c|c|}
\hline & $\begin{array}{c}\mathrm{MSi} \\
\mathrm{g} / \mathrm{kg} \mathrm{P}^{0,75}\end{array}$ & $\begin{array}{l}\text { Matières } \\
\text { sèches }\end{array}$ & $\begin{array}{c}\text { Matières } \\
\text { organiques }\end{array}$ & $\begin{array}{l}\text { Matières } \\
\text { azotćes* }\end{array}$ & $\begin{array}{l}\text { Cellulose } \\
\text { Weende }\end{array}$ & $\begin{array}{c}\text { MOd } \\
\text { g/kg MS }\end{array}$ & $\begin{array}{c}\text { MAd } \\
\mathrm{g} / \mathrm{kg} \mathrm{MS}\end{array}$ \\
\hline OIDU & $8 \pm \pm 3$ & $59,6 \pm 0,9$ & $61,8 \pm 1,0$ & $65,3 \pm 0,8$ & $29,6 \pm 2,3$ & $567 \pm 9$ & $87 \pm 1$ \\
\hline OMU & $87 \pm 2$ & $59,4 \pm 0,6$ & $61,9 \pm 0,6$ & $67,0 \pm 0,6$ & $35,6 \pm 0,9$ & $577 \pm 5$ & $89 \pm 1$ \\
\hline $\mathrm{OMD}_{1} \mathrm{U}$ & $87 \pm 3$ & $59,2 \pm 0,5$ & $61,6 \pm 0,5$ & $65,6 \pm 0,4$ & $30,5 \pm 2,6$ & $57 \pm \pm 5$ & $78 \pm 1$ \\
\hline $\mathrm{OMD}_{2} \mathrm{U}$ & $86 \pm 3$ & $59,3 \pm 0,5$ & $61,9 \pm 0,5$ & $64,6 \pm 0,9$ & $34,6 \pm 0,7$ & $578 \pm 4$ & $83 \pm 1$ \\
\hline
\end{tabular}
tibles.

MSi : matière sèche ingérée; MOd : matière organique digestible; MAd : matières azotées diges-

* OMU - OIDU et $\mathrm{OMD}_{1} \mathrm{U} P<0,05 ; \mathrm{OMU}-\mathrm{OMD}_{2} \mathrm{U} \mathrm{P}<0,01$.

Les coefficients d'utilisation digestive (CUD) de la matière sèche (MS) et de la matière organique (MO) des rations globales sont très normaux (respectivement $59,2$ à 59,6 et $6 \mathrm{I}, 6$ à $6 \mathrm{I}, 9)$; le maximum d'écarts respectifs enregistrés entre les régimes s'élève à 0,4 et 0,3 points. Les différences sont légèrement plus fortes entre les CUD de l'azote : $67,0 \pm 0,6$ avec 1'orge mûre non traitée (OMU) contre 65,6 $\pm 0,4$ après traitement faible $\left(\mathrm{OMD}_{1} \mathrm{U}\right)$ et $64,6 \pm 0,9$ après traitement fort $\left(\mathrm{OMD}_{2} \mathrm{U}\right)$, alors que le CUD de l'orge immature déshydratée (OIDU) est de 65,3 $\pm 0,8$.

Seul le CUD de l'azote du régime OMU est, soit significativement $(\mathrm{P}<0,05$ pour $\mathrm{OIDU}$ et $\left.\mathrm{OMD}_{1} \mathrm{U}\right)$, soit très significativement $\left(\mathrm{P}<\right.$ o,oI pour $\left.\mathrm{OMD}_{2} \mathrm{U}\right)$ plus élevé que ceux des 3 autres régimes. Ces derniers ne sont, par contre, pas significativement différents entre eux. Il en résulte que par $\mathrm{kg}$ de $\mathrm{MS}$, le régime à base d'orge mûre, qui a subi le traitement hydrothermique fort, renferme $6 \mathrm{~g}$ de MAd de moins $(83 \mathrm{~g})$ que cette même orge non traitée $(89 \mathrm{~g})$; cette différence est hautement significative, alors qu'elle n'est que de $2 \mathrm{~g}(87 \mathrm{~g})$ pour les deux autres régimes.

En revanche, les CUD de la matière organique oscillant entre $6 \mathrm{I}, 6$ et $6 \mathrm{I}, 9$, le contenu en énergie digestible (MOd) de tous les régimes est pratiquement similaire. Les valeurs extrêmes, non significativement différentes, sont comprises entre 567 土 $9 \mathrm{~g}(\mathrm{OIDU})$ et 578 土 $4 \mathrm{~g}\left(\mathrm{OMD}_{2} \mathrm{U}\right)$ par $\mathrm{kg}$ de MS. 
b) Bilan de l'utilisation métabolique de l'azote (tab1. 6).

Le niveau énergétique (MOd/ $\mathrm{P}^{0,75}$ ) est quasiment identique avec tous les régimes: $47,7 \pm \mathrm{I}, 5 \mathrm{~g}$ à $5^{\circ} \pm \mathrm{I}, 3 \mathrm{~g}$; par contre, celui d'azote digestible ( $\left.\mathrm{Nda} / \mathrm{P}^{0,75}\right)$ et le rapport entre ces deux éléments $(\mathrm{Nda} / \mathrm{MOd})$ sont très significativement plus faibles $(\mathrm{P}<\mathrm{o}, \mathrm{OI})$ pour le régime à l'orge mûre traitée à haute température $\left(\mathrm{OMD}_{2} \mathrm{U}\right)$, cela par suite de la forte chute du CUD de l'azote de ce régime.

\section{TABLEAU 6}

Bilan et utilisation métabolique de l'azote $\pm s m$

\begin{tabular}{|c|c|c|c|c|c|c|c|}
\hline & $\operatorname{MOd}^{*} / \mathrm{P}^{20,75}$ & $\mathrm{Nda}{ }^{*} / \mathrm{P}^{0,75}$ & $\mathrm{Nda} / \mathrm{MOd}$ & $\underset{\mathrm{P}^{0,75}}{N \text { bilan/ }}$ & $\frac{\mathrm{I}-\mathrm{F}-\mathrm{U}}{\mathrm{I}-\mathrm{F}} \frac{\mathrm{C}(\mathrm{CR})}{} \times 100$ & $\frac{\mathrm{I}-\mathrm{F}-\mathrm{U}}{\mathrm{I}(\mathrm{CUP})} \times 100$ & $\begin{array}{c}\text { Pds } \\
\text { moy. } \\
(\mathrm{kg})\end{array}$ \\
\hline OIDU & $47,7 \pm 1,5$ & $1,17 \pm 0,04$ & $2,46 \pm 0,03$ & $0,44 \pm 0,04$ & $37,2 \pm 3,2$ & $24,4 \pm 2,2$ & 255 \\
\hline OMU & $50,0 \pm 1,3$ & $1,24 \pm 0,00$ & $2,48 \pm 0,00$ & $0,45 \pm 0,03$ & $36,3 \pm 2,4$ & $24,3 \pm 1,5$ & 257 \\
\hline $\mathrm{OMD}_{1} \mathrm{U}$ & $49,8 \pm 1,0$ & $1,20 \pm 0,00$ & $2,42 \pm 0,00$ & $0,37 \pm 0,03$ & $31,0 \pm 3,1$ & $20,4 \pm 2,1$ & 259 \\
\hline $\mathrm{OMJ}_{2} \mathrm{U}$ & $49,6 \pm 1,5$ & $1,14 \pm 0,00$ & $2,30 \pm 0,00$ & $0,33 \pm 0,01$ & $29,3 \pm 2,1$ & $18,9 \pm 1,3$ & 258 \\
\hline
\end{tabular}

* MOd : matière organique digestible ; Nda : azote digestible apparent ; 1 : $\mathrm{N}$ ingéré ; I : $\mathrm{N}$ fécal ; $\mathrm{U}$ : $\mathrm{N}$ urinaire

CUP : OIDU $\left.-\mathrm{OMD}_{2} \mathrm{U} \mathrm{P}<0,01 ; \mathrm{OMU}-\mathrm{OMJ}\right)_{2} \mathrm{U} \mathrm{P}<0,05$.

$N$ bilan $/ t^{10,75}:$ OIDU $-\mathrm{OMD}_{2} \mathrm{UP}<0,01$; OMD $-\mathrm{OMD}_{2} \mathrm{U} \mathrm{P}^{\mathrm{P}}<0,01$; OMD $-\mathrm{OMD}_{1} \mathrm{UP}<0,05$.

Les coefficients de rétention azotée ( $\mathrm{N}$ retenu/ $\mathrm{N}$ digéré ou $\mathrm{CR}$ ) respectifs des régimes $\mathrm{OIDU}, \mathrm{OMU}, \mathrm{OMD}_{1} \mathrm{U}$ et $\mathrm{OMD}_{2} \mathrm{U}$, sont de $37,2 \pm 3,2 ; 36,3 \pm 2,4 ; 3 \mathrm{I}, \mathrm{O}$ $\pm 3, \mathbf{I}$ et $29,3 \pm 2, \mathrm{I}$ et ceux des CUP. ( $\mathrm{N}$ retenu/ $\mathrm{N}$ ingéré) de $24,4 \pm 2,2 ; 24,3$ $\pm \mathrm{I}, 5 ; 20,4 \pm 2, \mathrm{I}$ et $\mathrm{I} 8,9 \pm \mathrm{I}, 3$. On remarque que le CR et le CUP du régime à base d'orge fortement chauffée sont nettement plus faibles que ceux à l'orge mûre non traitée ou à l'orge immature déshydratée. Cette baisse hautement significative atteint I9 $\mathrm{p}$. Ioo pour le $\mathrm{CR}(\mathrm{P}<0,05)$ et $22 \mathrm{p}$. Ioo pour le CUP $(\mathrm{P}<0,02)$. I,es valeurs corresponđantes de l'orge mûre faiblement chauffée ne sont, par contre, pas significativement différentes d'aucun des trois autres régimes.

Les bilans azotés rapportés au poids métabolique $\left(\mathrm{P}^{\mathbf{0}, 75}\right)$ confirment encore plus nettement (tabl. 6) cette tendance défavorable au traitement hydrothermique fort de l'orge.

\section{2. - Niveau d'ingestion et croissance (tabl. 7)}

Les quantités insuffisantes d'orge immature déshydratée nous ont obligés à arrêter l'expérimentation de ce régime au 68 e jour ; par contre, pour les 3 autres régimes, il a été possible de poursuivre la comparaison $I_{5}$ jours de plus, soit donc pendant 83 jours. 
Le nombre limité de données individuelles ( 4 animaux par régime) et la durée par trop courte de l'essai confèrent à nos résultats une simple valeur indicative.

Le niveau de matière sèche ingérée ad libitum a été semblable avec les trois régimes à orge mûre : $94-97 \mathrm{~g} / \mathrm{kg} \mathrm{P}^{0,75}$ et légèrement plus fort : $103 \mathrm{~g} / \mathrm{kg} \mathrm{P}^{0,75}$ pour le régime à 1'orge immature (consommation de paille de litière exceptée et non estimée).

TABLEAU 7

Niveau d'ingestion et croissance

\begin{tabular}{|c|c|c|c|c|c|c|c|c|}
\hline & \multirow{2}{*}{$\begin{array}{c}\text { Durée } \\
\text { de l'essai (j) } \\
\text { et nbre } \\
\text { d'animaux }\end{array}$} & \multirow{2}{*}{$\begin{array}{c}\text { Pds } \\
\text { initial } \\
(\mathrm{kg})\end{array}$} & \multirow{2}{*}{$\begin{array}{l}\text { l'ds } \\
\text { final } \\
(\mathrm{kg})\end{array}$} & \multirow{2}{*}{$\begin{array}{c}\text { Gain moyen } \\
(\mathrm{g} / \mathrm{j})\end{array}$} & \multirow{2}{*}{$\begin{array}{l}\text { kg MISi \% } \\
\text { kg pds vif } / \mathrm{j}\end{array}$} & \multirow{2}{*}{$\begin{array}{l}\operatorname{kg} \text { MOd } \% \\
\text { kg pds vif } / \mathrm{j}\end{array}$} & \multicolumn{2}{|c|}{ Indices de consomm } \\
\hline & & & & & & & $\begin{array}{l}\mathrm{MSi} / \mathrm{kg} \\
\text { de gain }\end{array}$ & $\begin{array}{l}\mathrm{UF} / \mathrm{kg} \\
\text { de gain }\end{array}$ \\
\hline OIDU & $68-3$ & 311 & 415 & $\begin{array}{c}1539 \\
(1500-1 \quad 618)\end{array}$ & 2,36 & 1,321 & 5,6 & 3,2 \\
\hline $\mathrm{OMU}$ & $83-4$ & $29 y^{\prime}$ & $4 \geq 1$ & $\left(\begin{array}{c}1540 \\
1343-1919\end{array}\right)$ & 2,16 & 1,183 & 5,0 & 3,1 \\
\hline $\mathrm{OMD}_{1} \mathrm{U}$ & $83-4$ & 295 & 405 & $\begin{array}{c}1329 \\
\left(\begin{array}{ll}1 & 096(6-1627)\end{array}\right)\end{array}$ & 2,20 & 1,270 & 6,0 & 3,5 \\
\hline $\mathrm{OMD}_{2} \mathrm{U}$ & $83-4$ & 308 & 120 & $\begin{array}{c}1367 \\
\left(\begin{array}{cc}1 & 169-1506\end{array}\right)\end{array}$ & 2,26 & 1,239 & 5,9 & 3,6 \\
\hline
\end{tabular}

* Selon formule Leroy $:$ UF/kg MS $=\frac{(\mathrm{MOd}+1,25 \mathrm{MG} d) \times 3,6-1000}{188,3}$.

Les gains moyens journaliers enregistrés dans 1a séquence de poids 294-42I $\mathrm{kg}$ pendant les 83 jours de mesure, atteignent : I $540 \mathrm{~g}$ avec le régime d'orge mûre non traitée (OMU), I $329 \mathrm{~g}$ avec la même orge mûre traitée à basse température $\left(\mathrm{OMD}_{1} \mathrm{U}\right)$ et $\mathrm{I} 367 \mathrm{~g}$ avec celle traitée à température élevée $\left(\mathrm{OMD}_{2} \mathrm{U}\right)$. Les différences respectives en faveur de l'orge non traitée ressortent à +2 II et + I $73 \mathrm{~g} /$ jour, soit de 1'ordre de I2 p. Ioo.

I'indice de consommation exprimé en matière sèche (MS) ou en unités fourragères par $\mathrm{kg}$ de gain est de II à I2 p. Ioo plus faible dans le lot orge mûre non traitée.

Le groupe à l'orge immature déshydratée (OIDU) a réalisé durant les 68 jours d'essai un gain journalier et un indice de consommation identiques à ceux du groupe recevant l'orge mûre non traitée.

\section{DISCUSSION}

Parmi les nombreuses opinions formulées au sujet des répercussions du traitement hydrothermique d'une céréale sur sa valeur nutritive et son efficacité pour la croissance des bovins, deux sont diamétralement opposées. En effet, selon certains, 
la cuisson (KojIma et al., I968; GARRET et al., I968) ou la gélatinisation (MUDD et. PERRY, I969) exercent des effets défavorables; d'autres, par contre, observent une influence améliorante sensible sur la croissance ou la valeur énergétique du mais (MATSUShIma, I966; BÉRANGER et al., I972) ot du sorgho (HALE et al., Ig66 et $\mathrm{HALE}_{\mathrm{L}}$ I967). En réalité, les actions thermiques peuvent différer d'un traitement à l'autre suivant sa nature, son mode d'application et la céréale traitée.

Les résultats obtenus avec notre traitement hydrothermique de l'orge mûre réhydratée à $36 \mathrm{p}$. Ioo, appliqué à la pression atmosphérique, et à faible $\left(57^{\left.-97^{\circ} \mathrm{C}\right) \text { ou }}\right.$ forte $\left(75^{-10} 7^{\circ} \mathrm{C}\right)$ température, infirment les deux opinions extrêmes : ce type de traitement n'accroît ni n'abaisse le contenu en énergie digestible, puisque les niveaux énergétiques et azotés et la forme de présentation des rations étaient identiques. La seule action négative notée surtout avec le traitement fort est la chute hautement significative du CUD des matières azotées et de leur disponibilité métabolique. Cette action négative se marque avec grande netteté au niveau du bilan azoté (CR ou CUP), du gain de poids et de l'indice de consommation, bien que la fraction d'amidon facilement attaquable par les $\alpha$-amylases microbiennes ait presque doublé avec le traitement fort.

La fraction d'amidon rapidement dégradable in vitro ne représente que Io,7 p. Ioo de la matière sèche dans l'orge mûre originelle non traitée ; le traitement thermique faible $\left(\mathrm{OMD}_{\mathbf{1}} \mathrm{U}\right)$ la fait passer à $\mathrm{I}_{3}, 2 \mathrm{p}$. IoO et le traitement fort $\left(\mathrm{OMD}_{2} \mathrm{U}\right)$ à $\mathrm{I} 8,5 \mathrm{p}$. Ioo. Cette dernière valeur est comparable à celle $(20,6 \mathrm{p}$. Ioo) notée dans notre première étude avec la récolte I 968 de la même variété d'orge immature, traitée dans des conditions analogues ; alors que celle de l’orge immature (OID) de la présente expérience est bien plus élevée (27,I p. Ioo) (tabl. I et fig. I).

De ce fait, le rapport de 6,7 existant dans l'orge mûre non traitée (OMU) entre cette fraction et 1'urée ajoutée (I6 $\mathrm{g} / \mathrm{kg}$ MS) passe après 1'application de la chaleur à 8,3 dans $O M D_{1} U$ et à $I I, 6$ dans $O M D_{2} U$; il atteint $I 5,9$ dans la même orge récoltée immature (OID). Ces deux derniers rapports sont donc proches de celui relevé pour 1'orge immature I968 (I5,9). La présente expérience confirme donc pleinement notre précédente observation montrant que le traitement hydrothermique accroît notablement la fraction d'amidon facilement dégradable à la $\alpha$-amylase et par voie de conséquence le rapport entre cette fraction et l'urée (ZEITER et al., I97I). Dès lors, l'on pouvait raisonnablement supposer qu'il en serait de même en ce qui concerne l'effet bénéfique de ce traitement à l'orge mûre associée à l'urée sur le bilan azoté et la croissance.

Or, nos données prouvent clairement un phénomène significativement inverse : bien que tous les régimes en comparaison aient été isoénergétiques (niveau matières organiques digestibles $\left./ \mathrm{P}^{0,75}\right)$, ceux à orge mûre traitée $\left(\mathrm{OMD}_{1} \mathrm{U}\right.$ et $\left.\mathrm{OMD}_{2} \mathrm{U}\right)$ se montrent moins efficaces que ceux à base đe la même orge mûre non traitée (ONU) et à orge immature déshyđratée (OIDU).

Ce résultat négatif s'expliquerait par les effets thermiques exercés sur les protéines de l'orge. En effet, l'analyse des caractéristiques physico-chimiques des protéines de l'orge immature et mûre, avant et après les traitements, fait ressortir clairement que celles-ci ont été sérieusement modifiées, surtout à haute température $\left(\mathrm{OMD}_{2}\right)$.

La composition en acides aminés indispensables de l'orge mûre semble avoir été peu affectée (tab1. 2) ; la plupart n'ont point diminué ; seules ont baissé plus ou moins 
les teneurs en thréonine et isoleucine $(-8 \mathrm{p}$. I0o), en histidine ( - Io p. Ioo) et surtout en lysine ( - I3,5 p. Ioo) dont la disponibilité (tabl. 3) a de surcroît été fortement affectée (-20 p. xoo). En outre, la solubilité de la fraction albumines + globulines, la plus riche en lysine, a décru de $66 \mathrm{p}$. Ioo et celle des prolamines de $63 \mathrm{p}$. Ioo; et cela quelle que soit la sévérité du traitement (tabl. 3).

Cette action dénaturante de notre traitement se manifeste également dans l'orge immature dont la teneur en humidité avant traitement était pourtant plus élevée $(57 \mathrm{p}$. I00). On note effectivement (tabl. 3) dans ce cas après déshydratation, une baisse de même ordre que pour l'orge mûre, de la teneur en lysine totale ( -2 I p. IOo) et en lysine disponible ( -24 p. IO0). Les concentrations de l'orge traitée en cet acide aminé semblent donc davantage influencées par l'intensité du chauffage que par l'état d'hydratation initiale.

Les teneurs en $\mathrm{N}$ soluble exprimées en pourcentages de $\mathrm{N}$ total étant voisines ( 36 à $40 \mathrm{p}$. Ioo) dans tous les régimes expérimentaux les différences de richesse en lysine disponible n'expliquent pas à elles seules les écarts observés dans l'efficacité azotée. Les causes résideraient donc sans doute ailleurs.

Dans le cas de l'orge mûre non traitée, deux facteurs ont vraisemblablement joué en faveur de l'efficacité du régime :

— près de 90 p. Ioo de 1'amidon natif de 1'orge sont dégradés par la micropopulation du'rumen en oses et holosides à courtes chaînes (Orskov, I969; THIVEND et JOURNET, I968) lui fournissant ainsi l'énergie nécessaire à sa protéinosynthèse à partir de l'azote uréique ;

- les protéines natives de l'orge sont fortement remaniées en protéines microbiennes dans le rumen (Orskov et al., I97 I ; DurAnd et al., I972). Cela laisse supposer que le remaniement ayant revalorisé les protéines de l'orge, le régime orge non traitée + urée a permis aux taurillons de satisfaire entièrement leurs besoins azotés quantitatifs et qualitatifs.

Dans le cas de l'orge traitée, il y a eu indubitablement une augmentation de la fraction amylacée facilement dégradable par les $\alpha$-amylases bactériennes (fig. I) ; ce qui aurait dû̀ en principe contribuer à une meilleure utilisation de l'urée par les microorganismes de la panse. En revanche, en raison des dénaturations provoquées par notre traitement hydrothermique (tabl. 3), les protéines de l'orge semblent d'après l'étude toute récente de DURAND et al. (I972) transiter le rumen sans avoir été remaniées par sa micropopulation. Comme de surcroît l'orge apporte plus de 60 p. roo de l'azote total des régimes et que le traitement expérimenté a entraîné en outre une chute non négligeable de la digestibilité de cet azote, il est très vraisemblable que la protéinosynthèse microbienne n'était pas parvenue, malgré un niveau MAd élevé, à compenser entièrement les déficiences qualitatives des matières azotées des régimes à orge traitée et cela d'autant moins que le chauffage était plus intense. Ces faits pourraient donc expliquer la plus faible efficacité azotée des régimes $\mathrm{OMD}_{1} \mathrm{U}$ et $\mathrm{OMD}_{2} \mathrm{U}$, d'autant plus que le traitement hydrothermique appliqué a plus spécialement affecté la lysine, facteur limitant primaire de l'orge, et essentiel pour la croissance. Les résultats de croissance le confirment d'ailleurs, puisque malgré un niveau d'énergie digestible relativement plus élevé (MOd), les groupes qui ont consommé les rations à base d'orge mûre traitée accusent un gain de poids sensiblement plus faible (tabl. 7). Il semble donc bien que les matières azotées de ces régimes possédaient une efficacité biologique plus basse que celles du régime à base d'orge mûre non traitée. 
Somme toute, sur le plan strictement nutritionnel, le traitement hydrothermique que nous avons étudié, d'une orge mûre destinée au jeune taurillon, se révèle non seulement inutile, mais encore manifestement contre-indiqué en cas de supplémentation par de l'urée, en raison de l'amoindrissement de la valeur biologique des protéines qu'il entraîne. En serait-il de même pour le maïs? Cette question fait l'objet d'une étude en cours.

Du point de vue appliqué, nos résultats de niveau de consommation et de gain de poids réalisés en alimentation individuelle ad libitum par des taurillons normands maintenus sur litière de paille consommée à volonté, suggèrent, malgré leur nombre limité, que 1'emploi d'une ration condensée formée d'orge mûre 60 p. Ioo associée à sa propre paille (35 p. roo), supplémentée uniquement avec I,6 p. Ioo d'urée et 4 p. roo d'un mélange minéral vitaminisé correct, est susceptible d'assurer des gains de poids journaliers élevés, du moins à partir d'un poids vif proche de $300 \mathrm{~kg}$.

Cependant, l'avantage de ce type de régime, qui supprime le recours à des tourteaux onéreux, demanderait à être confirmé par une expérimentation en lots dans des conditions pratiques, d'élevage sur un bien plus grand nombre d'animaux.

Reçu pour publication en mars 1972.

\section{REMERCIEMENTS}

Les auteurs expriment leurs vifs remerciements à Odette SzYLIT, pour les tests à l' $\alpha$-a mylase, à G. Viroben, pour l'analyse des protéines, à J.-P. Melcion et M. Fradin pour la conduite des traitements thermiques.

\section{SUMMARY}

\section{EFFEC'T OF HEAT MOISTURE TREATMEN'T OF A IMMATURE AND RIPE BARLEY ON I'TS NU'TRITIVE VALUE AND ON UREA UTILIZATION IN YOUNG BULLS}

In a previous experiment (ZELTER et al., I97I) we have shown that treatment of immature barley with moist heat increased the amount of starch which can be hydrolysed with $\alpha$-amylase and improved the value of added urea for the young growing bull. The present study was carried out to determine if the same treatment of ripe barley after it had been rehydrated had the same beneficial effects.

One lot of ripe barley was subdivided and treated as follows : A no treatment (control);

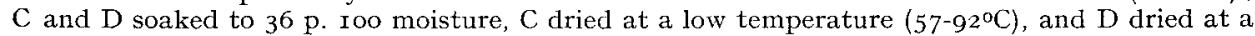
higher temperature $\left(75^{-10} 7^{\circ} \mathrm{C}\right)$. One other lot of barley $(\mathrm{B})$ was harvested immature from the same field as that which was allowed to ripen ; this immature barley was dried under the same conditions as the barley sample $D$.

Each of the four lots of barley were mixed with barley straw (35 p. Ioo), a mineral vitamin supplement, and urea which provided $34-36 \mathrm{p}$. I oo of the total nitrogen in the ration. The four diets : $O M U(A), O M D_{1} U(C), O M D_{2} U(D)$ and $O I D U(B)$ were isoenergetic and iso-nitrogenous, and were fed as cubes to four groups of Norman bulls which were between 6 and Io months old.

After a preliminary period, the digestibilities of the major constituents were determined, along with the coefficients of utilization of the nitrogen ( $\mathrm{N}$ retained/ $\mathrm{N}$ digested and $\mathrm{N}$ retained/ $\mathrm{N}$ ingested) in two successive ${ }_{5}$ days balance periods (see experimental outline). Finally the level of ingestion and the weight gain of the same animals individually fed ad libitum were measured for I 2 weeks. 
The heat treatment of the ripe barley increased the fraction of easily hydrolysed starch by $23 \mathrm{p}$. I 100 and $72 \mathrm{p}$. Ioo for the mild and strong treatment respectively (fig. I and table I).

Neither of the two treatments affected the digestibility of the organic matter (table 5). They decreased significantly, for the strong heat treatment, the digestibility coefficient of the nitrogen (64.6 compared with 67 in the control) and the nitrogen retention coefficient $(29.3 \pm 2.1$ compared with $36.3 \pm 2.4$ ) (table 6).

The weight gains were high for all groups but they were higher and the feed conversion ratios were lowest for the untreated ripe barley and for the dried immature barley (table 7 ).

These unfavourable effects of the two moist heat treatments of ripe barley are undoubtedly due to denaturation of the protein, indicated particularly by the lower levels of essential amino acids and by the lower availability of lysine (tables 2 and 3 ). Thus it seems possible that there is a deficiency in the available protein and amino acids which had not been compensated by the bacterial proteinosynthesis from urea.

Therefore, the use of this type of treatment is not to be recommanded for ripe barley.

\section{RÉFÉRENCES BIBIIOGRAPHIQUES}

Béranger C., Thivend P., Jarrige R., I972. Influence des traitements mécaniques et hydrothermiques sur la valeur nutritive du maïs pour les jeunes bovins à l'engrais. Ann. Zootech., 21 I 75-I9o.

DURAND M., 1970. Influence $d u$ traitement technologique de l'aliment énergétique (orge immature) sur le métabolisme de ses glucides et de l'urée dans le rumen du mouton. C. R. Acad. Sci., 217 (Série D) 2167-2I70.

Durand M., Ben Ameur M., Viroben G., I972. Influence du traitement hydrothermique de l'orge sur la digestion de ses glucides et de ses protéines, en l'absence et en présence d'une source complémentaire d'azote (urée ou soja) (sous presse).

Garret W. N., Lofgreen G. T., Hull R. L., I968. Steam pressure processed sorghum grain for steers. J. Anim. Sci., 27, I 164 (Abst. I65).

Guilbot A., Mercier Ch., rg62. Répercussion sur la digestibilité de l'amidon des modifications de sa structure physico-chimique au cours de ses transformations technologiques. Ind. Agric. Alim., 79, 939-947.

Hale W. H., Cuitun L., Saba W. J., Taylor B., Theurer B., ig66. Effect of steam processing and Flaking milo and barley on performance and digestion by steers. J. Anim. Sci, 25, 392-396.

HALE W. H., r96\%. Effect of moist heat treatment of cereal grains on growth, feed utilization by cattle. Feedstuffs, 39, no 9, 29.

Kojima Y., Nakai S., Kawashima R., Uesakas, ig68. Effect of cooked barley or milo mixed to the ration on its digestibility. Japan. J. Zoot. Sci., 39, 543-548.

Matsushima J. K., 1966. Greatest feed efficiency from flaked corn. Feedstuffs, 38, no $4,29$.

Mudd C. A., Perry T. W., 1969. Raw craked VS, expanded gelatinized corn for beef cattle. J. Anim. Sci., 28, 822-826.

Orskov E. R., 1969. Post ruminal digestion of carbohydrates : extent and implications. Rev. Cubana Cienc. Agric., 3, I-13.

Orskov E. R., Fraser C., McDonald I., I97I. Digestion of concentrates in sheep. 3. Effects of rumen fermentation of barley and maize diets on protein digestion. Brit. J. Nutr., 26, 477-486.

Roach A. C., Sanderson P., Williams D. R., i967. Comparison of methods for the determination of available value in animal and vegetable protein sources. J. Sci Fd. Agric., 18, 274-278.

Thivend P., Journet M., r968. Utilisation digestive de l'amidon de l'orge chez le ruminant. Ann. Biol. anim. Bioch. Biophys., 8, 449-45 I.

Zelter S.-Z., Charlet-Léry G., Tisserand J.-L., i971. Influence chez le taurillon en croissance du traitement de conservation (ensilage ou déshydratation) de la céréale immature (orge, maîs) sur sa valeur nutritive et sur l'efficacité métabolique de l'urée ajoutée. Ann. Zootech., 20, I35-r52. 